\title{
Can venous base excess replace arterial base excess as a marker of early shock and a predictor of survival in trauma?
}

Ramesh $\underline{\text { Wijaya }}^{1}$, MBBS, FRCSE, Jia Hui $\underline{\mathrm{Ng}}^{2}$, MBBS, MRCSE, Lester $\underline{\text { Ong }^{3}}$, MBBS, MRCSE, Andrew Siang Yih Wong ${ }^{1}$, MBBS, FRCSE

INTRODUCTION Arterial base excess is an established marker of shock and predictor of survival in trauma patients. However, venous blood is more quickly and easily obtained. This study aimed to determine if venous base excess could replace arterial base excess as a marker in trauma patients at presentation and if venous base excess is predictive of survival at 24 hours and one week.

METHODS This was a prospective study of 394 trauma patients presenting to the emergency department of a tertiary hospital over a 17-month period. Data on base excess at presentation, vital signs, shock index (SI), injury severity score (ISS), and mortality at 24 hours and one week was collected and analysed.

RESULTS Arterial and venous blood gas tests were performed on 260 and 134 patients, respectively. Patients were stratified into groups based on their SI and ISS for analysis. There was no statistical difference between mean venous blood gas and arterial blood gas levels at presentation when $\mathrm{SI}>0.7$, regardless of ISS $(p>0.05)$. The mortality rate was $4.57 \%$. Both venous and arterial base excess was lower in nonsurvivors compared to survivors $(p<0.05)$. However, at 24 hours and one week, the difference in base excess values at presentation between survivors and nonsurvivors was greater when using venous base excess compared to arterial base excess ( 11.53 vs. 4.28 and 11.41 vs. 2.66 , respectively). CONCLUSION In conclusion, venous base excess can replace arterial base excess in trauma patients as a means of identifying and prognosticating early shock.

Keywords: arterial, base excess, survival, trauma, venous

\section{INTRODUCTION}

The use of vital signs alone may be inadequate in the resuscitation of trauma patients due to the presence of compensatory physiologic mechanisms in early shock. Scalea et al found that up to $80 \%$ of critically ill patients who are normotensive and have adequate urine output may remain in a state of compensated shock. ${ }^{(1)}$ As such, the use of adjunctive biochemical markers of shock to assess tissue hypoxia and prevent underresuscitation in a timelier manner has been investigated. Arterial base excess has emerged as an important marker in assessing resuscitation, as well as prognosticating trauma patients. ${ }^{(2-4)}$ Numerous authors have correlated arterial base excess with ongoing haemorrhage, ${ }^{(5)}$ eventual development of multiple organ failure ${ }^{(6,7)}$ and mortality. ${ }^{(8,9)}$

However, venous blood is more easily obtained during the establishment of venous access, an essential early step in trauma management. Therefore, the use of venous base excess as a marker reduces the number of procedures performed in a busy trauma resuscitation. It also avoids the inherent risks of performing an arterial puncture. Venous base excess has been shown to correlate well with arterial base excess in critically ill, ${ }^{(10-12)}$ mechanicallyventilated trauma ${ }^{(13)}$ and acute trauma patients. ${ }^{(14-16)}$ In addition, it has been suggested that venous base excess better reflects overall tissue perfusion and is therefore a better predictor of severity of shock and mortality. ${ }^{(17)}$
As it has not been established if venous base excess can replace arterial base excess in acute trauma, Schmelzer et al undertook a prospective study in 2007 that compared venous and arterial base excess values in acute trauma patients; the study found that venous base excess, but not arterial base excess, was predictive of survival at 24 hours. ${ }^{(4)}$ However, correlation with survival after a longer interval was not studied.(4) The present study aimed to determine if venous base excess could replace arterial base excess in a trauma patient at presentation. We compared venous and arterial base excess values of acute trauma patients, and analysed the predictive value of venous versus arterial base excess for survival at 24 hours and one week.

\section{METHODS}

We conducted a single-centre prospective study at a tertiary hospital in Singapore with a dedicated trauma service. All acute trauma patients presenting at our institution's emergency department between January 2009 and May 2010 were included. Trauma patients were identified based on our institution's trauma activation criteria (Appendix). According to the institution's trauma management protocol, all trauma patients should have their base excess values measured at presentation, and at six and 24 hours after presentation. The decision to have either arterial or venous base excess measurement taken at presentation was made at the discretion of the trauma team leader. If the arterial

${ }^{1}$ Department of General Surgery, Changi General Hospital, ${ }^{2}$ Department of Otolaryngology, ${ }^{3}$ Department of General Surgery, SingHealth, Singapore Correspondence: Dr Ramesh Wijaya, Associate Consultant, Department of General Surgery, Changi General Hospital, 2 Simei Street 3 , Singapore 529889. ramesh_wijaya@cgh.com.sg 
base excess measurement was taken at presentation, venous base excess would not be measured, and vice versa. Peripheral blood samples were also taken. All patients without arterial or venous base excess measurements taken at presentation were excluded. We collected data prospectively, including patient demographics, arterial or venous base excess values at presentation, shock index (SI), injury severity score (ISS), and mortality at 24 hours and one week.

$\mathrm{SI}$ is defined as heart rate divided by systolic blood pressure. It has been shown to be useful in identifying early shock and correlates with mortality. ${ }^{(18-20)}$ Patients were divided into groups with Sls of $\leq 0.7$ ('nonshock' group), > 0.7 to 1 ('early shock' group) and $>1$ ('shock' group) based on previous study data. ${ }^{(18,19,21)}$ The ISS was developed by Baker et al in 1974 as a scalar measure of all anatomical injuries sustained during trauma. ${ }^{(22)}$ It is the sum of the squares of the highest Abbreviated Injury Scale grade (developed by the Association for the Advancement of Automotive Medicine) in each of the three most severely injured body regions. The ISS has been validated to correlate with mortality and a score $>15$ is defined as major trauma. ${ }^{(23)}$ Patients were divided into two groups; ISS score $\leq 15$ (designated as the non-major trauma group) and ISS score of $>15$ (designated as the major trauma group).

The patients who had venous or arterial base excess values taken at presentation were stratified into three and two groups based on their SI and ISS scores, respectively. We compared the distribution of patients into various SI and ISS score strata in the venous base excess group versus the arterial base excess group using chi-square test. Two-sample $t$-tests were performed for each stratum to determine if base excess from arterial and venous samples yielded similar results. The arterial and venous base excess values of nonsurvivors versus survivors were also analysed via the two-sample $t$-tests to determine if either could be predictive of survival at 24 hours and one week. A p-value of $<0.05$ was considered statistically significant. All analyses were performed using Stata version 10.1 (StataCorp, College Station, TX, USA).

\section{RESULTS}

A total of 485 patients fulfilled our institution's trauma activation criteria. Of these patients, 91 were excluded as their blood gas levels were not measured on presentation. The mean age of the patients was 36.2 years and $324(82.2 \%)$ were men. The mean SI score was 0.720 and the mean ISS score was 10.6. Of the 394 patients finally included in the study, 134 (34.0\%) had venous base excess performed on arrival, while the rest of patients had arterial base excess performed. The characteristics of the study population are summarised in Table I.

For our analysis, we stratified patients in the venous and arterial base excess groups according to their SI and ISS scores (Table II). A comparison of the venous and arterial base excess groups found no statistically significant difference between the various SI and ISS strata ( $p>0.05)$. This allowed us to conclude that there was no significant difference in severity of shock and injury between the patients in the venous and arterial base excess groups, before proceeding to further analysis.
Table I. Characteristics of the trauma patients $(n=394)$.

\begin{tabular}{lr}
\hline Characteristic & No. (\%) \\
\hline Age* (yr) & $36.2(9-91)$ \\
Gender & \\
$\quad$ Male & $324(82.2)$ \\
$\quad$ Female & $70(17.8)$ \\
Shock index & \\
$\leq 0.7$ & $255(64.7)$ \\
$>0.7-1$ & $112(28.4)$ \\
$>1$ & $27(6.9)$ \\
Injury severity score & \\
$\leq 15$ & $283(71.8)$ \\
$>15$ & $111(28.2)$ \\
Type of blood gas analysis at presentation & \\
$\quad$ Arterial & $260(66.0)$ \\
$\quad$ Venous & $134(34.0)$ \\
\hline
\end{tabular}

*Data presented as mean (range).

Table II. Venous and arterial base excess stratified by shock index (SI) and injury severity score (ISS).

\begin{tabular}{lcc}
\hline Parameter & \multicolumn{2}{c}{ Base excess } \\
\cline { 2 - 3 } & Venous $(\mathbf{n}=\mathbf{1 3 4})$ & Arterial $(\mathbf{n}=\mathbf{2 6 0})$ \\
\hline SI & $77(57.5)$ & $178(68.5)$ \\
$\leq 0.7$ & $46(34.3)$ & $66(25.4)$ \\
$>0.7-1$ & $11(8.2)$ & $16(6.2)$ \\
$>1$ & & $186(71.5)$ \\
ISS & $97(72.4)$ & $74(28.5)$ \\
$\leq 15$ & $37(27.6)$ & \\
$>15$ & &
\end{tabular}

Data presented as no. (\%).

We analysed the mean venous and arterial base excess groups stratified by SI (Table II). The mean venous base excess measurements were $-0.35,-0.82$ and -6.82 in the 'nonshock', 'early shock' and 'shock' groups, respectively. The mean arterial base excess measurements for the corresponding SI groups were $-1.32,-1.85$ and -6.91 , respectively. Statistical tests showed that the difference between the venous and arterial base excess values in the 'nonshock' group was significant $(p=0.034)$, although there were no significant differences between the mean venous and arterial base excess values in the 'early shock' and 'shock' groups ( $p=0.073$ and $p=0.961$, respectively) (Table III).

In the analysis of the data stratified by ISS, the mean venous base excess value was -0.37 in the non-major trauma group and -2.81 in the major trauma group. Comparatively, the mean arterial base excess value was -0.96 and -3.92 in the non-major and major trauma groups, respectively. The differences between mean venous and arterial base excess values in either ISS group was not statistically significant ( $p>0.05$, Table III). A total of eight patients did not survive to 24 hours. An additional ten patients did not survive to one week. The overall mortality rate at one week was $4.57 \%(n=18)$. Table IV shows the mean venous and arterial base excess values of the survivors compared to that of the nonsurvivors. At both 24 hours and one week, both arterial and venous base excess values were significantly lower among the nonsurvivors compared to the survivors $(p<0.05)$. The difference 
Table III. Comparison of mean venous and arterial base excess values stratified by shock index (SI) and injury severity score (ISS).

\begin{tabular}{lccc}
\hline Parameter & \multicolumn{2}{c}{ Base excess } & p-value \\
\cline { 2 - 3 } & Venous & Arterial & \\
\hline SI & & \\
$\leq 0.7$ & $-0.35 \pm 2.35$ & $-1.32 \pm 3.70$ & 0.034 \\
$>0.7-1$ & $-0.82 \pm 2.99$ & $-1.85 \pm 2.95$ & 0.073 \\
$>1$ & $-6.82 \pm 4.70$ & $-6.91 \pm 4.97$ & 0.961 \\
ISS & & & \\
$\leq 15$ & $-0.37 \pm 2.57$ & $-0.96 \pm 3.48$ & 0.121 \\
$>15$ & $-2.81 \pm 4.27$ & $-3.92 \pm 3.92$ & 0.175 \\
\hline
\end{tabular}

Data presented as mean \pm standard deviation. Two-sample $t$-tests were used.

Table IV. Mean venous and arterial base excess values of the survivors and nonsurvivors $(n=394)$.

\begin{tabular}{lcc}
\hline Parameter & \multicolumn{2}{c}{ Base excess } \\
\cline { 2 - 3 } & & Arterial \\
\hline At $\mathbf{2 4}$ hr & $-0.87 \pm 3.01$ & $-1.70 \pm 3.77$ \\
$\quad$ Survivors $(n=386)$ & $-12.40 \pm 0.57$ & $-5.98 \pm 5.03$ \\
Nonsurvivors $(n=8)$ & $<0.001$ & 0.007 \\
p-value & & \\
At 1 wk & $-0.79 \pm 2.86$ & $-1.65 \pm 3.72$ \\
Survivors $(n=376)$ & $-12.20 \pm 0.49$ & $-4.31 \pm 4.93$ \\
Nonsurvivors $(n=18)$ & $<0.001$ & 0.009 \\
p-value & & \\
\hline
\end{tabular}

Data presented as mean \pm standard deviation. Two-sample $t$-tests were used.

was more marked in terms of venous base excess values. The mean difference in venous base excess values at presentation between survivors and nonsurvivors at 24 hours was 11.53, whereas the difference in arterial base excess values in these two groups of patients was lower at 4.28. Similarly, the mean difference in venous base excess values at presentation between survivors and nonsurvivors at one week was 11.41, whereas the difference in arterial base excess values in these two groups of patients was lower at 2.66.

\section{DISCUSSION}

Many prior studies have shown that arterial and venous base excess demonstrate good correlation. ${ }^{(10,12,13,15,16)}$ However, Rudkin et $\mathrm{al}^{(16)}$ also pointed out that despite the good correlation between paired arterial and venous base excess values in trauma patients, the $95 \%$ limits of agreement were unacceptably wide in $20 \%$ of the patients. Our study approached this clinical question from a different angle. We compared arterial and venous base excess values between groups of patients in the same SI and ISS stratum, rather than in the same patient. This approach would aid us in identifying specific groups of patients for whom arterial and venous base excess values are interchangeable. No prior study to date has stratified trauma patients in this manner to investigate the correlation between arterial and venous base excess.

Our results show that when patients were stratified by ISS, there was no significant difference in patients' arterial and venous base excess values within the ISS $\leq 15$ and ISS $>15$ groups $(p>0.05)$. This is the first study to suggest that venous and arterial base excess values are comparable within the different ISS stratification groups. In addition, there was no significant difference between arterial and venous base excess values when SI was > 0.7-1 ('early shock' group) and when SI was $>1$ ('shock' group). However, when SI was $\leq 0.7$ ('nonshock' group), arterial base excess showed significantly lower values than venous base excess $(p=0.034)$. Although venous and arterial base excess results do not appear to be comparable in this group of patients, some studies have suggested that venous base excess may be superior, as discussed below.

Arterial base excess is more widely accepted as a surrogate laboratory marker of shock in trauma patients, although there are studies that suggest that when arterial and venous base excess values do not correlate, venous base excess is a better marker of early changes in tissue perfusion. Rudkin et al proposed that this may be because postcapillary acid-base status better reflects tissue hypoperfusion. ${ }^{(16)}$ Schmelzer et al ${ }^{(4)}$ proposed that the lung's ability to readily exchange carbon dioxide and oxygen might mean that arterial blood gases may not demonstrate the hypercarbia that exists in venous blood. As such, venous blood gases may better reflect tissue perfusion, while arterial blood gases better reflect the adequacy of ventilation and oxygenation. ${ }^{(4)}$ In addition, Oropello et al monitored arterial and venous blood gas continuously in an animal model of haemorrhagic shock and found that changes in venous blood gas levels occurred earlier and were more significant than changes in arterial blood gas levels. ${ }^{(17)}$

Arterial base excess has been used to prognosticate trauma patients at presentation. ${ }^{(6)-9)}$ Therefore, this study further investigated the utility of venous base excess in comparison with arterial base excess as a predictor of mortality in trauma patients. The results show that both arterial and venous base excess values at presentation were significantly lower in nonsurvivors compared to survivors $(p<0.05)$ at 24 hours and one week. However, the difference was greater for venous base excess values, suggesting that venous base excess is not inferior to arterial base excess at predicting survival of trauma patients at 24 hours and one week. In concordance with the results of our study, Schmelzer et al ${ }^{(4)}$ studied the survival of 100 trauma patients at 24 hours and found that while the mean venous base excess value was significantly lower in nonsurvivors than survivors $(-2.9$ vs. $-0.3, p=0.004)$, the difference in arterial base excess value was not significant.

We were unable to obtain paired arterial and venous blood gas samples from our study population. Therefore, we could not study the 95\% limits of agreement between arterial and venous base excess, a statistic that was reported by other similar studies on this topic. Furthermore, the decision to have an arterial or venous blood sample taken at presentation was made at the discretion of the trauma team leader. It can be argued that this may introduce bias to our results; therefore, we performed an analysis of the proportion of patients in the SI and ISS strata to ensure that there was no significant difference in the SI and ISS of patients who had an arterial or venous blood gas sample taken.

The strength of our study is that data was collected prospectively. In comparison to similar studies on this topic, the present study included among the largest number of trauma 
patients and investigated the ability of venous base excess to predict survival not only at 24 hours, but also one week, which was not investigated by other similar studies.

Venous base excess shows similar results to arterial base excess in patients with more than early shock $(\mathrm{SI}>0.7)$, regardless of severity of injury as measured by the ISS. It also appears to be comparable to arterial base excess in predicting mortality at both 24 hours and one week. Venous base excess may therefore be a viable alternative to arterial base excess for assessing and prognosticating trauma patients at presentation.

\section{REFERENCES}

1. Scalea TM, Maltz S, Yelon J, et al. Resuscitation of multiple trauma and head injury: role of crystalloid fluids and inotropes. Crit Care Med 1994; 22:1610-5.

2. Husain FA, Martin MJ, Mullenix PS, Steele SR, Elliott DC. Serum lactate and base deficit as predictors of mortality and morbidity. Am J Surg 2003; 185:485-91.

3. Kincaid EH, Miller PR, Meredith JW, Rahman N, Chang MC. Elevated arterial base deficit in trauma patients: a marker of impaired oxygen utilization. J Am Coll Surg 1998; 187:384-92.

4. Schmelzer TM, Perron AD, Thomason MH, Sing RF. A comparison of central venous and arterial base deficit as a predictor of survival in acute trauma. Am J Emerg Med 2008; 26:119-23.

5. Davis JW, Shackford SR, MacKersie RC, Hoyt DB. Base deficit as a guide to volume resuscitation. J Trauma 1988; 28:1464-7.

6. Davis JW, Parks SN, Kaups KL, Gladen HE, O'Donnell-Nicol S. Admission base deficit predicts transfusion requirements and risk of complications. J Trauma 1996; 41:769-74.

7. Sauaia A, Moore FA, Moore EE, et al. Early predictors of postinjury multiple organ failure. Arch Surg 1994; 129:39-45.

8. Rutherford EJ, Morris JA Jr, Reed GW, Hall KS. Base deficit stratifies mortality and determines therapy. J Trauma 1992; 33:417-23.

9. Siegel JH, Rivkind Al, Dalal S, Goodarzi S. Early physiologic predictors of injury severity and death in blunt multiple trauma. Arch Surg 1990; 125:498-508.

10. Middleton P, Kelly AM, Brown J, Robertson M. Agreement between arterial and central venous values for $\mathrm{pH}$, bicarbonate, base excess, and lactate. Emerg Med J 2006; 23:622-4.

11. Treger R, Pirouz S, Kamangar N, Corry D. Agreement between central venous and arterial blood gas measurements in the intensive care unit. Clin J Am Soc Nephrol 2010; 5:390-4.

12. Zakrison T, McFarlan A, Wu YY, Keshet I, Nathens A. Venous and arterial base deficits: do these agree in occult shock and in the elderly? A BlandAltman analysis. J Trauma Acute Care Surg 2013; 74:936-9.

13. Malinoski DJ, Todd SR, Slone S, Mullins RJ, Schreiber MA. Correlation of central venous and arterial blood gas measurements in mechanically ventilated trauma patients. Arch Surg 2005; 140:1122-5.

14. Arnold TDW, Miller M, van Wessem KP, Evans JA, Balogh ZJ. Base deficit from the first peripheral venous sample: a surrogate for arterial base deficit in the trauma bay. J Trauma 2011; 71:793-7.

15. Gindi M, Sattler S, Kevin Hoos, Matei M, Paulus C, Yens D. Can venous blood gas samples replace arterial blood gas samples for measurement of base excess in severely injured trauma patients? N Y Med J [online] 2007; 2. Available at: http://newyorkmedicaljournal.org/1/Archives/Sattler9-07.htm. Accessed June 8, 2015.

16. Rudkin SE, Kahn CA, Oman JA, et al. Prospective correlation of arterial vs venous blood gas measurements in trauma patients. Am J Emerg Med 2012; 30:1371-7.

17. Oropello JM, Manasia A, Hannon E, Leibowitz A, Benjamin E. Continuous fiberoptic arterial and venous blood gas monitoring in hemorrhagic shock. Chest 1996; 109:1049-55.

18. Cannon CM, Braxton CC, Kling-Smith M, et al. Utility of the shock index in predicting mortality in traumatically injured patients. J Trauma 2009; 67:1426-30.

19. McNab A, Burns B, Bhullar I, Chesire D, Kerwin A. A prehospital shock index for trauma correlates with measures of hospital resource use and mortality. Surgery 2012; 152:473-6.

20. Mutschler M, Nienaber U, Münzberg M, et al. The Shock Index revisiteda fast guide to transfusion requirement? A retrospective analysis on 21,853 patients derived from the TraumaRegister DGU. Crit Care 2013; 17:R172.

21. Birkhahn RH, Gaeta TJ, Terry D, Bove JJ, Tloczkowski J. Shock index in diagnosing early acute hypovolemia. Am J Emerg Med 2005; 23:323-6.

22. Baker SP, O'Neill B, Haddon W Jr, Long WB. The Injury Severity Score: a method for describing patients with multiple injuries and evaluating emergency care. J Trauma 1974; 14:187-96.

23. Copes WS, Champion HR, Sacco WJ, Lawnick MM, Keast SL, Bain LW. The Injury Severity Score revisited. J Trauma 1988; 28:69-77.

\section{APPENDIX}

Table I. Trauma activation criteria at our institution.

\begin{tabular}{|c|c|c|}
\hline Mechanism & Anatomical injury & Physiologic conditions \\
\hline $\begin{array}{l}\text { - Fall from a height }(6 \mathrm{~m} / 20 \mathrm{ft}) \\
\text { - Prolonged entrapment/extrication } \\
\text { (> } 20 \mathrm{~min}) \\
\text { - High-speed auto crash } \\
\text { - Ejection from vehicle } \\
\text { - Pedestrian road accident } \\
\text { - Assault }\end{array}$ & $\begin{array}{l}\text { - Penetrating injury (head, neck, torso) } \\
\text { - Pelvic fracture (excluding isolated femoral } \\
\text { neck fractures in elderly patients) } \\
\text { - Two or more long bone fractures } \\
\text { - Flail chest } \\
\text { - Proximal amputation } \\
\text { - Paralysis }\end{array}$ & $\begin{array}{l}\text { - Compromised airway } \\
\text { - Respiratory rate }<10 / \mathrm{min} \text { or }>30 / \mathrm{min} \\
\text { - Systolic blood pressure }<90 \mathrm{mmHg} \\
\text { and Glasgow Coma Scale score }<14\end{array}$ \\
\hline
\end{tabular}

